\title{
ANNOUNCEMENT / COMMUNIQUÉ
}

\section{Introducing our new look}

The Entomological Society of Canada is pleased to launch a new design for The Canadian Entomologist with the first issue for 2005. Although the primary purpose of the redesign is to modernize our Journal to meet the changing needs and desires of our readers and authors, it also incorporates contemporary typographic standards. The cover better reflects the diversity of research in entomology and related disciplines in Canada today.

We hope that our readers and subscribers will be pleased with the dramatic new design.

Richard A. Ring

Editor-in-Chief

\section{Notre nouvelle image}

La Société d'entomologie du Canada est heureuse de faire le lancement de la nouvelle présentation de sa revue, The Canadian Entomologist, dans ce premier numéro de l'année 2005. Bien que l'objectif principal de ce renouveau soit de moderniser la revue et de satisfaire les aspirations et les besoins changeants de nos lecteurs et de nos auteurs, nous lui avons donné par la même occasion une facture typographique moderne. La page couverture reflète mieux la diversité de la recherche en entomologie et dans les disciplines connexes du Canada contemporain.

Nous osons croire que nos lecteurs et nos abonnés seront ravis de ce design renouvelé et percutant.

Richard A. Ring

Directeur scientifique en chef 